\title{
Mecatrónica aplicada a la ingeniería de rehabilitación
}

Paola Andrea Niño S.*

Óscar Fernando Avilés $S$.**

\section{Resumen}

En este artículo se presenta una metodología de diseño integral y recurrente que aplicada a la Ingeniería de Rehabilitación, lo que dá como resultado el desarrollo de dispositivos de alta calidad, confiabilidad y seguridad para ser aplicados en la terapéutica, específicamente en la rehabilitación, y/o en medicina deportiva.

Se presentan tres desarrollos de sistemas mecatrónicos para aplicaciones en ingeniería de rehabilitación: un exoesqueleto para rehabilitación motora de miembro superior, silla de ruedas multifuncional y el desarrollo de una mano robótica de características antropomórficas.

Estos desarrollos son producto del trabajo interdisciplinario entre profesionales de la salud, de ingeniería y usuarios de dispositivos de rehabilitación, que de manera integral han colaborado con los integrantes del grupo de investigación DAVINCI para obtener los resultados aquí presentados.

Palabras clave: ingeniería de rehabilitación, diseño óptimo, mecatrónica.

\section{Mecatronics applied to rehabilitation engineering}

\section{Abstract}

In this article a design methodology that applies engineering principles to the design, modification, customization and/or fabrication of assistive technology for persons with disabilities is presented.

Three mechatronics systems are introduces an exoskeleton that allows exercises for three different joints of the arm at the same time, an mechatronics design protocol for development a prototype of a multifunctional wheelchair, that offers greater comfort, versatility and the possibility of making therapeutic

\footnotetext{
Ingeniera Electrónica, Magíster en Ingeniería Biomédica, Doctora en Ciencias de la Ingeniería-área Mecatrónica. Docente Asociada, Universidad Militar Nueva Granada. paola.polita@gmail.com

** Ingeniero Electrónico, Magíster en Sistemas Automáticos de Producción, Doctor en Ingeniería Mecánica en el área de Mecánica de sólidos y proyecto mecánico. Docente asociado, Universidad Militar Nueva Granada.
} 
exercises and a hand that has anthropomorphic measures and it's designed to look like a human hand in appearance and functionality. This works were developed by an interdisciplinary group leaded by the research group DAVINCI.

Key words: rehabilitation engineering, mechatronics engineering.

\section{Introducción}

La Ingeniería Mecatrónica se define como la integración sinérgica de la ingeniería mecánica, la ingeniería electrónica, la ingeniería de software y el control inteligente en el diseño y fabricación de productos y procesos industriales.

Los sistemas mecatrónicos están compuestos de partes mecánicas, eléctricas y electrónicas, dotadas de sensores que registran la información, microprocesadores que la interpretan, la procesan y la analizan y partes que reaccionan a esta información.

Como resultado de esta integración e interrelación nacen nuevos conceptos y tecnologías con el fin de propiciar la optimización de un producto o proceso industrial.

Un sistema mecatrónico posee, desde su concepción y diseño, características que lo identifican como un sistema óptimo tales como, alta confiabilidad, eficiencia, precisión, estabilidad, alto rendimiento, inteligencia y flexibilidad, entre otras.

Por otra parte, la Ingeniería de Rehabilitación en general es la aplicación de la ciencia y de la tecnología, con el objeto de atenuar las dificultades enfrentadas por individuos con discapacidades físicas, al efectuar las actividades de la vida diaria. La Ingeniería de Rehabilitación comprende el diseño de todos aquellos dispositivos que permitan aumentar o remplazar las funciones o estructuras de los sistemas vivos, cuando uno o más de ellas están deterioradas. Con esta definición, cualquier dispositivo, técnica o concepto utilizado en rehabilitación y que cuente con una base tecnológica, está dentro del alcance de la Ingeniería de Rehabilitación.

De tal forma, que si la Ingeniería de Rehabilitación es la aplicación de tecnología de punta en la solución de las necesidades de personas con algún tipo de limitación física o mental, se puede afirmar que la Ingeniería Mecatrónica provee los métodos de diseño y fabricación necesarios para obtener dispositivos que cumplan con las características de confiabilidad, funcionabilidad y seguridad necesarias para aplicaciones en rehabilitación.

Por lo tanto, la Ingeniería Mecatrónica es una herramienta fundamental en el desarrollo de la Ingeniería de Rehabilitación, ya que provee los conocimientos y herramientas necesarias para el desarrollo de sistemas integrales. En este artículo se presenta una breve definición del concepto de diseño mecatrónico y como este concepto ha sido aplicado a varios desarrollos en Ingeniería de Rehabilitación en dispositivos para rehabilitación motora, dispositivos para desplazamiento de personas con limitaciones de movimiento y desarrollo de manos robóticas.

\section{Diseño mecatrónico}

El diseño mecatrónico es una metodología que permite diseñar todo un prototipo a partir de la obtención de un modelo matemático que es llevado a un modelo computacional que permite, mediante la simulación de su comportamiento, determinar las características de fabricación como tipo de material a usar, estructura final, sistemas de actuación necesarios, sensores requeridos, sistema de control del sistema, en general es posible tener un prototipo final sin realizar su implementación física. Esta me- 
todología de diseño reduce costos de fabricación, de operación y optimiza el tiempo de diseño.

La interactividad en el proceso de diseño mecatrónico es un factor importante, existe una relación estrecha entre el modelo computacional que da la oportunidad de simular las características del sistema y el sistema físico que se implementará como prototipo final. En la figura 1 se puede observar un esquema que resume esta interacción.

El enfoque concurrente en este tipo de diseño permite desarrollar simultáneamente la estructura mecánica, el sistema de control y la etapa electrónica necesaria para integrar el sistema. En la figura 2 se observa un esquema de la metodología de diseño propuesta.

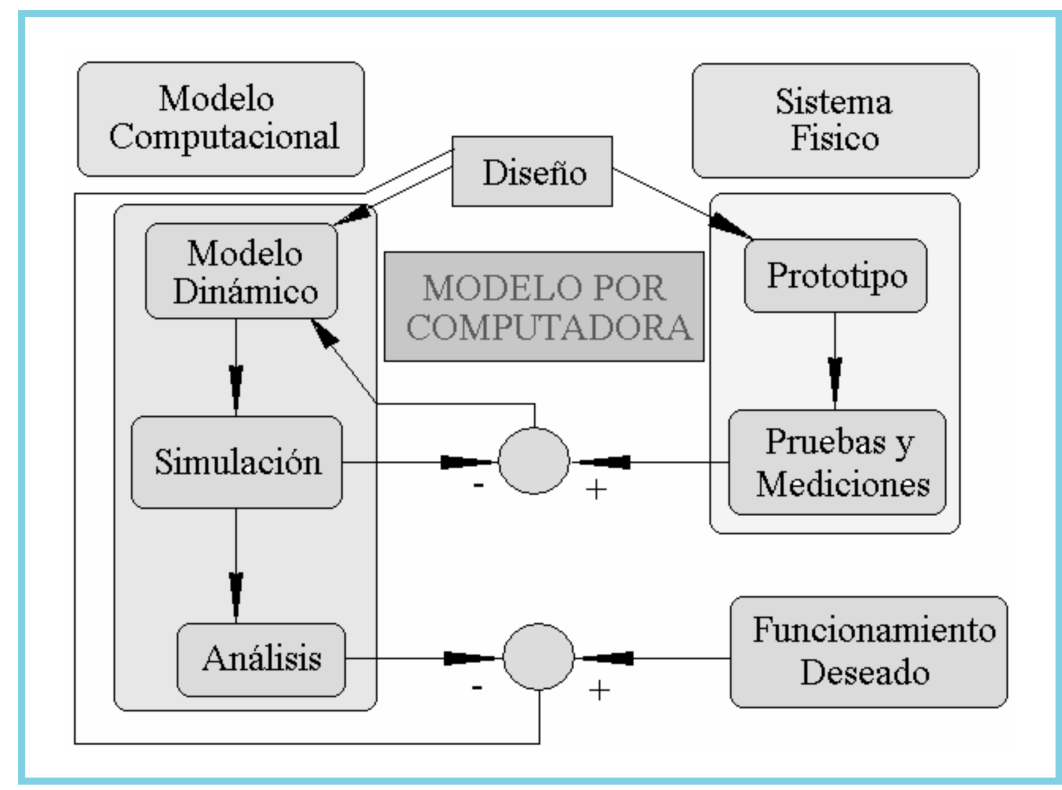

Figura 1. Interacción en el diseño mecatrónico

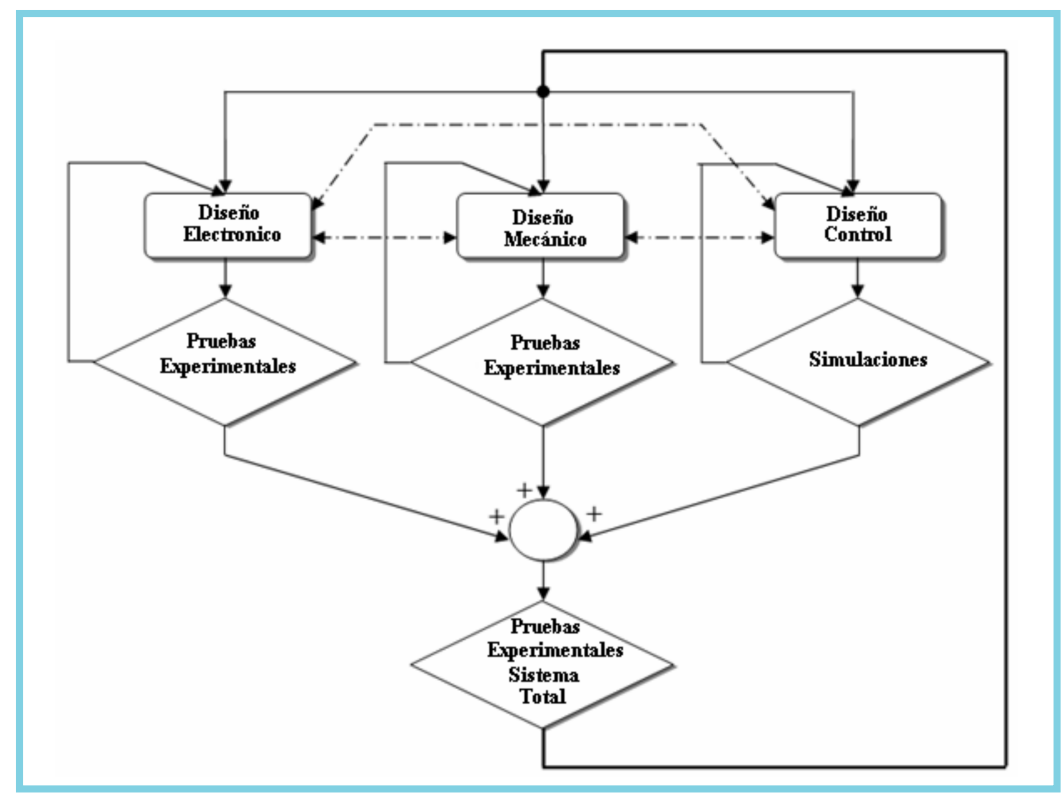

Figura 2. Metodología aplicada en el diseño mecatrónico 
En las siguientes secciones se presentan tres experiencias de desarrollo de dispositivos para Ingeniería de Rehabilitación a partir de utilizar la metodología aplicada en el diseño mecatrónico.

\section{Dispositivo para rehabilitación motora: exoesqueleto para rehabilitación motora}

El dispositivo de rehabilitación desarrollado es un exoesqueleto que se sujeta al brazo del paciente permitiéndole a éste realizar movimientos combinados, gracias a los tres grados de libertad que posee. El prototipo es controlado por medio de una interfaz de usuario que permite enviar órdenes de movimiento a la estructura desde un computador, el terapeuta modifica la terapia que debe realizar el paciente variando las trayectorias a seguir por el exoesqueleto.

Se puede programar la cantidad de repeticiones que el paciente debe realizar y almacenar los datos obtenidos de la posición de cada una de las articulaciones del miembro superior en cada sesión de terapia, para de esta forma monitorear el progreso del paciente. La posibilidad de llevar un control cronológico de la evolución del paciente es un beneficio adicional que brinda el dispositivo. El prototipo cumple la función de reeducación de movimientos (función pasiva), el fisioterapeuta realiza el movimiento con el dispositivo una vez y el exoesqueleto lo repite almacenando la rutina en su memoria, creando un banco de trayectorias programables por el especialista (1).

El diseño del prototipo se inició con el análisis biomecánico del brazo que permitió identificar los cinco grados de libertad del miembro superior. Estos grados de libertad son abducción y aducción del hombro, flexión y extensión del hombro, rotación interna y externa del húmero, flexión y extensión del codo, pronación y supinación de la mano. Continuando con el análisis se obtuvieron los centros de gravedad del miembro superior y a partir de estos datos se realizó un modelo en CAD, figura 3, con el fin de analizar su dinámica mediante la simulación de varios movimientos (2).
El análisis del modelo mecánico del brazo permitió obtener los torques máximos necesarios para mover cada articulación. Para la selección de los actuadores que mueven el exoesqueleto, era necesario analizar también la resistencia que puede ejercer el paciente al movimiento que el exoesqueleto induce y el peso propio del prototipo.
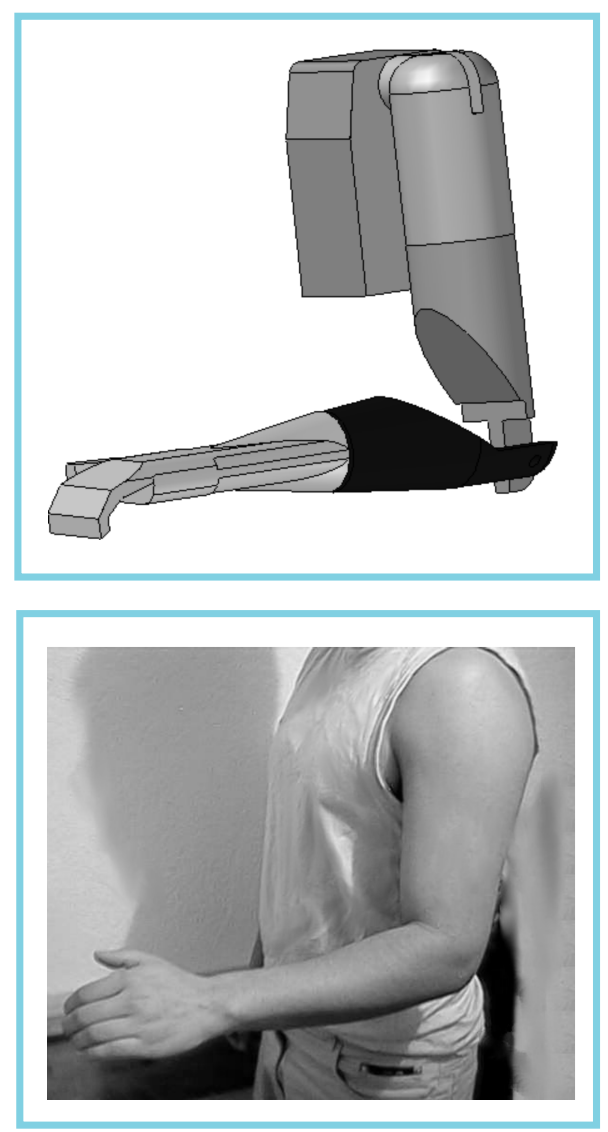

Figura 3. Modelo en CAD del miembro superior.

A partir de los datos obtenidos del análisis biomecánico de la extremidad superior, se establecieron los tres grados de libertad de la estructura que permiten la rotación interna-externa del húmero, la flexión-extensión del codo y la pronación-supinación de la muñeca, no se implementaron los movimientos para la aducciónabducción y flexión elevación del codo, por no considerarse necesarios para la fase inicial de desarrollo del dispositivo. El diseño en CAD se observa en la figura 4. 


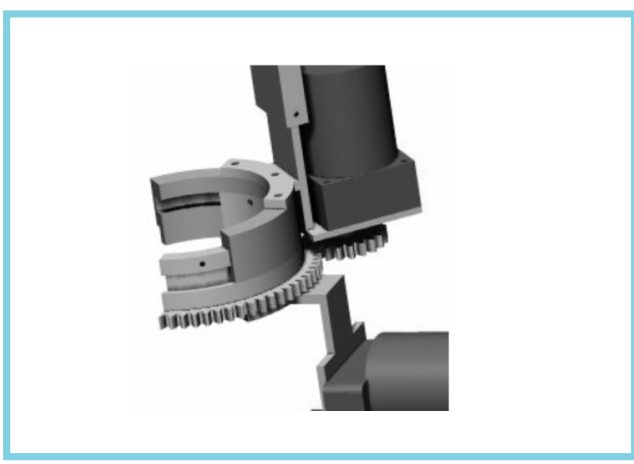

(a). Detalle férulas de rotación del húmero

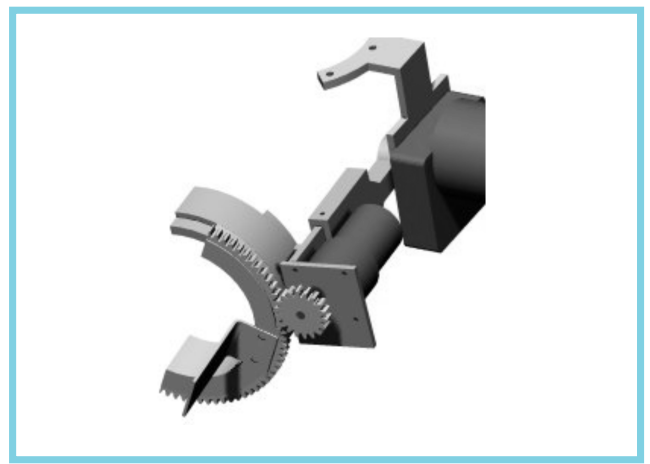

(c). Detalle mecanismo de prona y supina de la muñeca

Figura 4. Diseño en CAD del prototipo.

El CAD y CAE de la estructura básica del prototipo, (figura 5), permitió obtener los torques requeridos para mover cada grado de libertad dato necesario para la selección de los diferentes actuadores. También se realizó el análisis de elementos finitos de cada una de las piezas, usando

(a). FEM mecanismo rotación del húmero

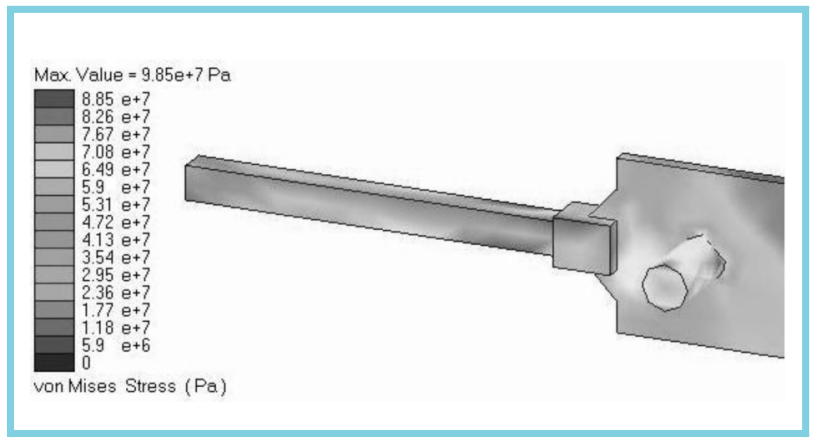

(c). FEM Soporte flexión hombro

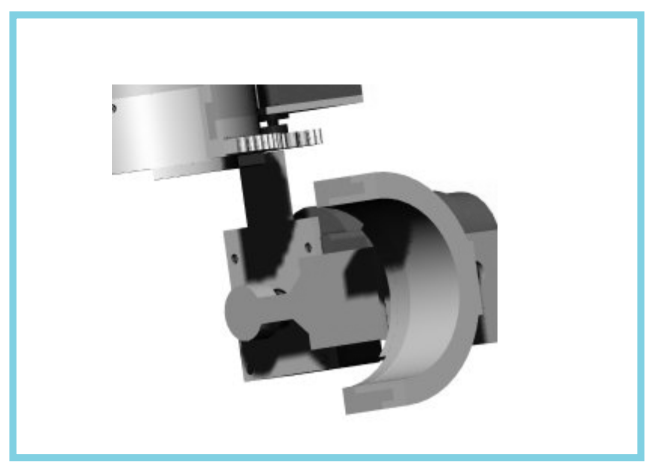

(b). Detalle férulas de flexión y extensión del hombro

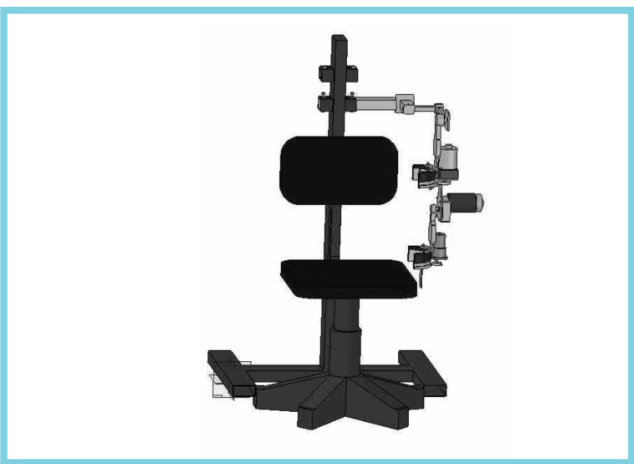

(d). Silla para paciente y soporte del prototipo

las propiedades mecánicas de diferentes materiales y aplicando las fuerzas obtenidas del análisis biomecánico, como se ilustra en la figura 6 . A partir de los resultados obtenidos, se seleccionó el aluminio como material para fabricar las piezas del exoesqueleto.

(b). FEM Soporte motor codo

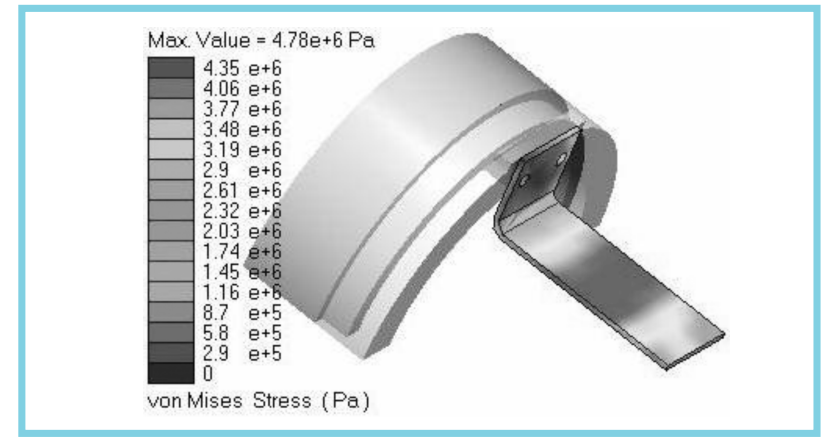

(d). FEM Férula mano

Figura 6. Resultado análisis por elementos finitos de distintas piezas. 
En la figura 7 se presenta el exoesqueleto colocado sobre un paciente. Para su operación se diseñó un sistema de control de posición que garantiza que los motores que hacen parte del exoesqueleto, sigan las trayectorias de las rutinas de rehabilitación programadas. El control tomó como entrada la posición en grados de cada una de las articulaciones del exoesqueleto y entrega como salida una señal PWM (Modulación por Ancho de Pulso) que por medio de una etapa de potencia maneja la operación de los actuadores.

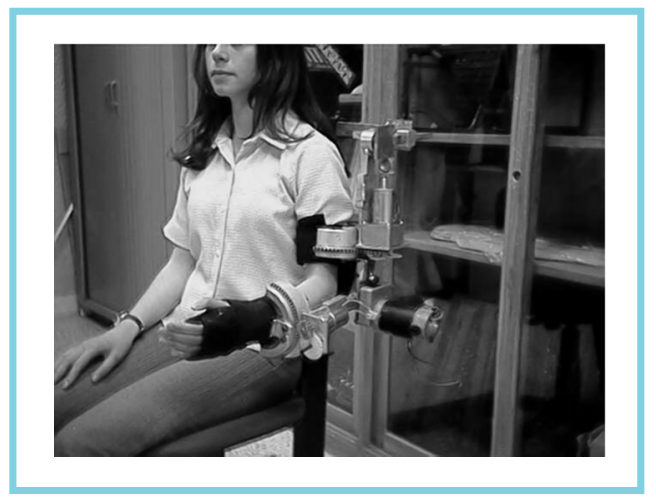

Figura 7. Exoesqueleto implementado sobre paciente.
El control se implementa a partir de inteligencia artificial, generando una base de reglas de lógica Fuzzy de acuerdo al esquema de la figura 8 (3).

Para programar las terapias se introduce la rutina a seguir con un modelo a escala, que posee los mismos grados de libertad que el exoesqueleto.

El terapeuta realiza las trayectorias a programar con este modelo, de tal forma que las trayectorias generadas son introducidas como la referencia del sistema de control, como se presenta en la figura 9.

Este tipo de programación (off-line) es especialmente útil al momento de realizar teleterapias, en donde el especialista introduce las terapias localmente y son ejecutadas de forma remota.

El programa de la interfaz de usuario está en capacidad de guardar los datos propios del paciente, así como puede guardar datos de una terapia y repetirlos.

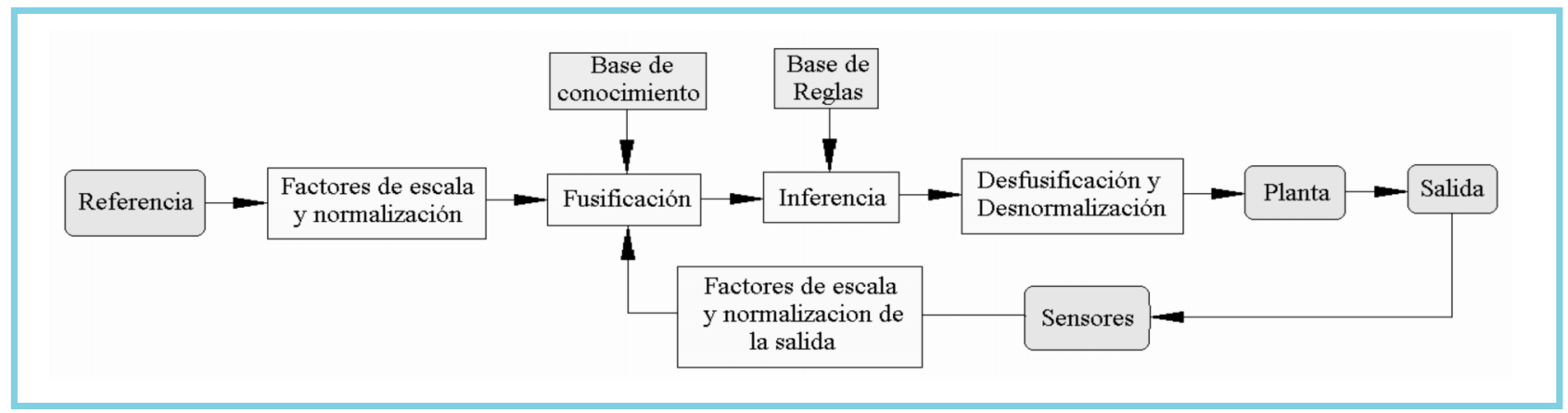

Figura 8. Sistema de control.

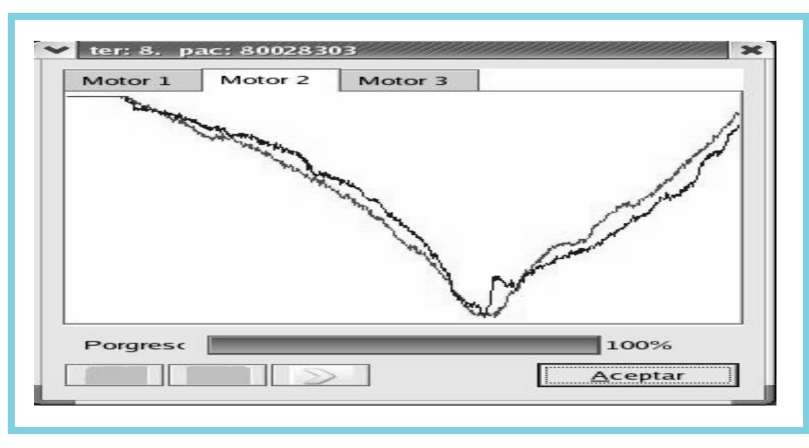

Figura 9. Interfaz de programación.
Las terapias realizadas son almacenadas en un archivo con el ánimo que el especialista las revise y analice si el paciente está respondiendo favorablemente a las terapias.

Es posible realizar terapias remotas con el exoesqueleto, esto ofrece la posibilidad de no desplazar al especialista hasta el lugar de ejecución de la terapia, puesto que esto es costoso y es difícil entrenar personal para realizar las terapias manualmente. 
De esta forma se presenta una nueva alternativa para rehabilitación, ofreciendo un exoesqueleto que permite la realización de ejercicios para tres articulaciones distintas al mismo tiempo.

A partir del diseño mecatrónico se obtuvo una estructura rígida, construida con materiales de alta resistencia, que cumple con las expectativas planteadas. El uso de herramientas CAD y CAE es importante en la minimización del tiempo empleado para ajustes. El esquema de control propuesto, desarrollado a partir de Lógica Difusa, cumple con los requerimientos de robustez y adaptabilidad que el sistema exige. El control del sistema se desempeña adecuadamente sin importar la variación del peso del paciente, proyectado entre 50 y $100 \mathrm{~kg}$.

\section{Dispositivo para desplazamiento: Silla de ruedas multifuncional}

La silla de ruedas supone una solución a las limitaciones de desplazamiento que tienen las personas con discapacidades físicas. Para los usuarios de silla de ruedas este es el medio por el cual pueden movilizarse y un dispositivo en el que pasan la mayor parte del día, es el elemento que va a permitir al usuario reintegrarse a su vida laboral y social. La silla de ruedas ha de cumplir, inicialmente, un doble requerimiento: por un lado, debe ser estable y cómoda; y por otro, debe permitir a su usuario un fácil desplazamiento.

De acuerdo a los diagnósticos médicos y terapéuticos el lograr estar de pie, así sea por pocos minutos al día, aporta significativos avances en los procesos de rehabilitación de pacientes con discapacidad en miembros inferiores. La calidad de vida de la persona con discapacidad mejora notablemente al realizar terapias que le permitan estar de pie por un intervalo de tiempo al día, ya que esta acción ayuda a la liberación de presión en zonas puntuales, a la normalización de las funciones del riñón y de la vejiga, al mejoramiento del sistema digestivo, al mantenimiento de la densidad del hueso, al mejoramiento de la flexibilidad y disminución de espasmos musculares, a mejorar la circulación y a mejorar la oxigenación de la sangre.
La silla desarrollada, tiene como finalidad ayudar al paciente en sus terapias de rehabilitación o en sus ejercicios de mantenimiento, porque le permite cambiar su postura y lo coloca de pie por un periodo de tiempo determinado o le permite acostarse completamente (5).

El diseño de una silla de ruedas debe tener como objetivo brindarle al usuario máxima funcionalidad, comodidad y movilidad. Para cumplir con este objetivo la silla debe ajustarse al individuo que la va a usar y no el usuario amoldarse a su silla. La selección y posterior utilización de una silla de ruedas no apropiada, puede resultar incomoda, causando problemas adicionales en la condición del paciente debido al esfuerzo continuo por modificar su postura (6).

Las medidas antropométricas indispensables para el diseño de la silla ofrecen la posibilidad de relacionarse íntimamente con la estatura del paciente. Razón por la cual es necesario realizar un análisis antropométrico con el fin de diseñar una silla de ruedas, para ello son útiles software de antropometría como el programa "Antroprojeto", realizado en la Universida de Federal de Juiz de Fora en Brasil, en este software se ingresa la estatura del individuo y se obtienen las medidas antropométricas de cuerpo de pies y en posición de decúbito, tal y como se aprecia en la figura 10.

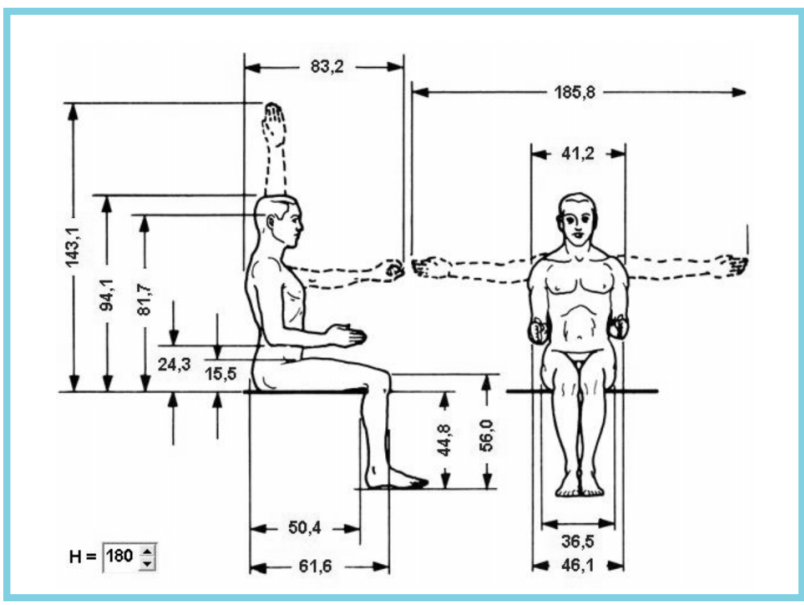

Figura 10. Medidas antropométricas del cuerpo obtenidas con el programa. 


\section{Antroprojeto}

Con los datos obtenidos de las consideraciones antropométricas y luego de realizar un análisis biomecánico de la posición ideal del usuario en la silla, se determinan las características de diseño como que el armazón debe ser una estructura rígida y resistente que sirva de apoyo a los motores que proporcionan el movimiento a la silla y a las ruedas giratorias que facilitan su maniobrabilidad, además el armazón debe permitir el pivoteamiento del sillín y dar soporte al actuador que da el movimiento de declinación. En general, a partir de estas consideraciones se realiza un bosquejo del armazón de la silla, como se observa en la figura 11.

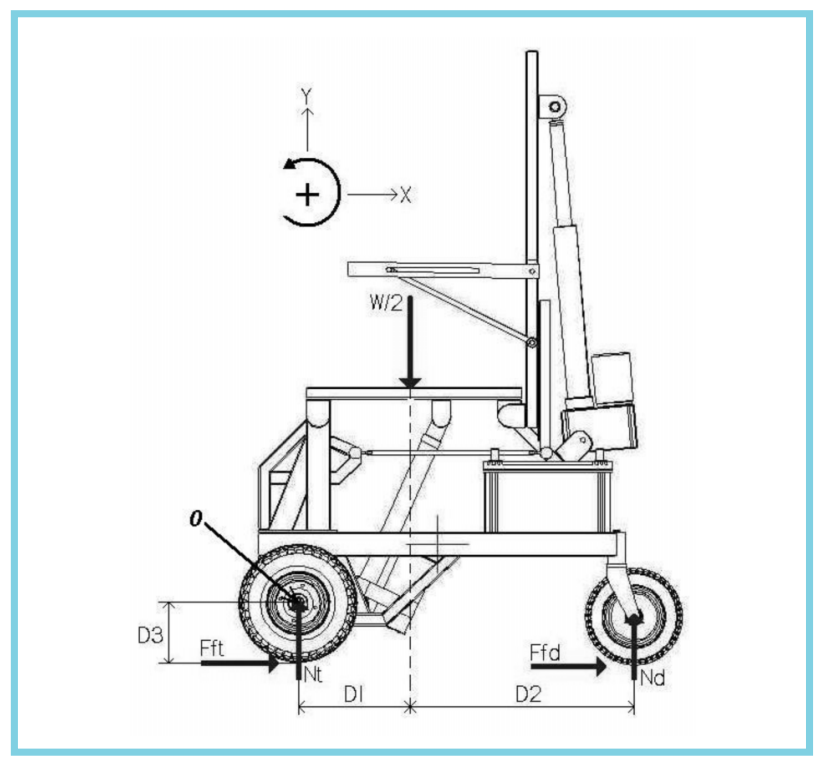

Figura 11. Diseño del armazón de la silla de ruedas.

El encargado de otorgar las características de potencia y robustez en su desplazamiento a la silla de ruedas es su motor de propulsión, por tal motivo su elección es de gran importancia.

Teniendo en cuenta lo anterior se seleccionó una base de potencia de acople directo, que por lo general es más durable y apta para transitar por terreno irregular, claro esta que este tipo de base de potencia comúnmente no desarrolla grandes velocidades con respecto a las bases de potencia guiadas.
La velocidad estándar en silla de ruedas es de $7 \mathrm{~K} \mathrm{~m} / \mathrm{h}$. Se seleccionó una rueda neumática de 10 pulgadas de diámetro exterior Dt, para cumplir con las especificaciones calculadas. Con los valores de velocidad y torque del motor se puede calcular la potencia que debe desarrollar el mismo para mover la silla de ruedas, esta es

$$
\mathrm{P}=\frac{\mathrm{TN}}{63030}=1.146 H P
$$

El sistema de declinación está conformado por un actuador eléctrico lineal, anclado al armazón por un soporte giratorio en un extremo y al sillín en el otro, con el fin de empujar o retraer el sillín respecto del armazón provocando el efecto de declinación.

El sillín se encuentra pivoteando sobre el armazón mediante unos rodamientos en A y B propiamente alineados, como se observa en la figura 12 (a).

Aislando el sillín, se muestran las reacciones en los rodamientos y la fuerza ejercida por el actuador, figura 12 (b).
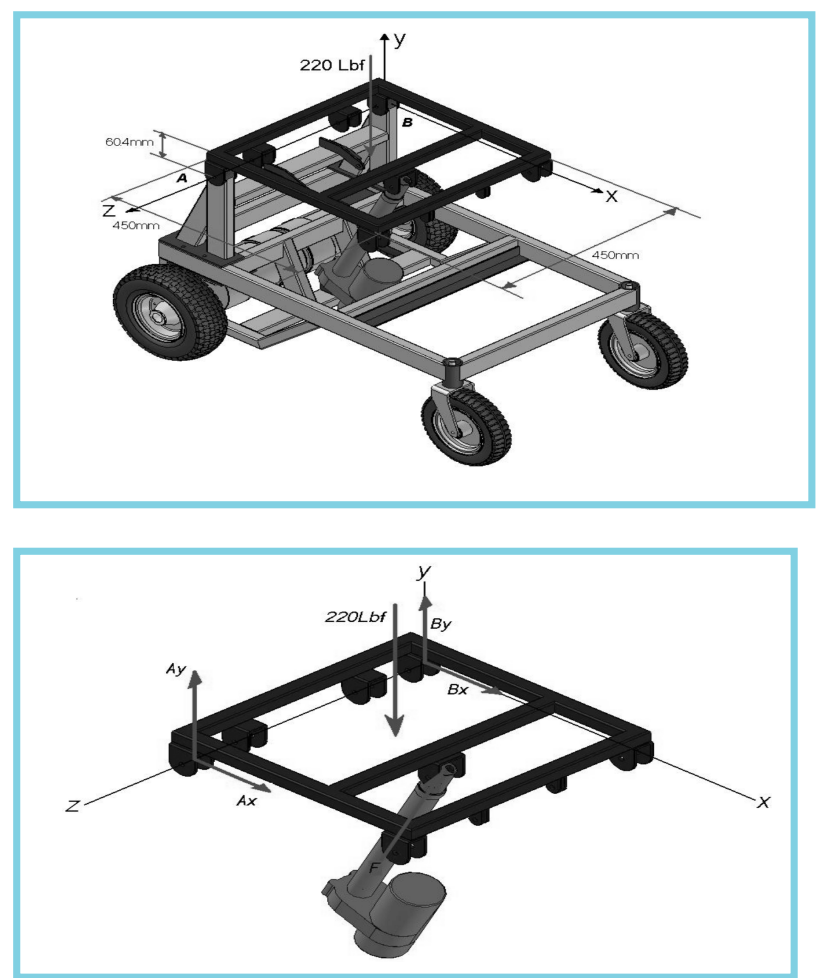

Figura 12. Sistema de declinación. 
El mecanismo de reclinación está formado por un actuador eléctrico lineal que permite el pivoteamiento del espaldar sobre el sillín provocando el efecto de reclinación, como se observa en la figura 13.
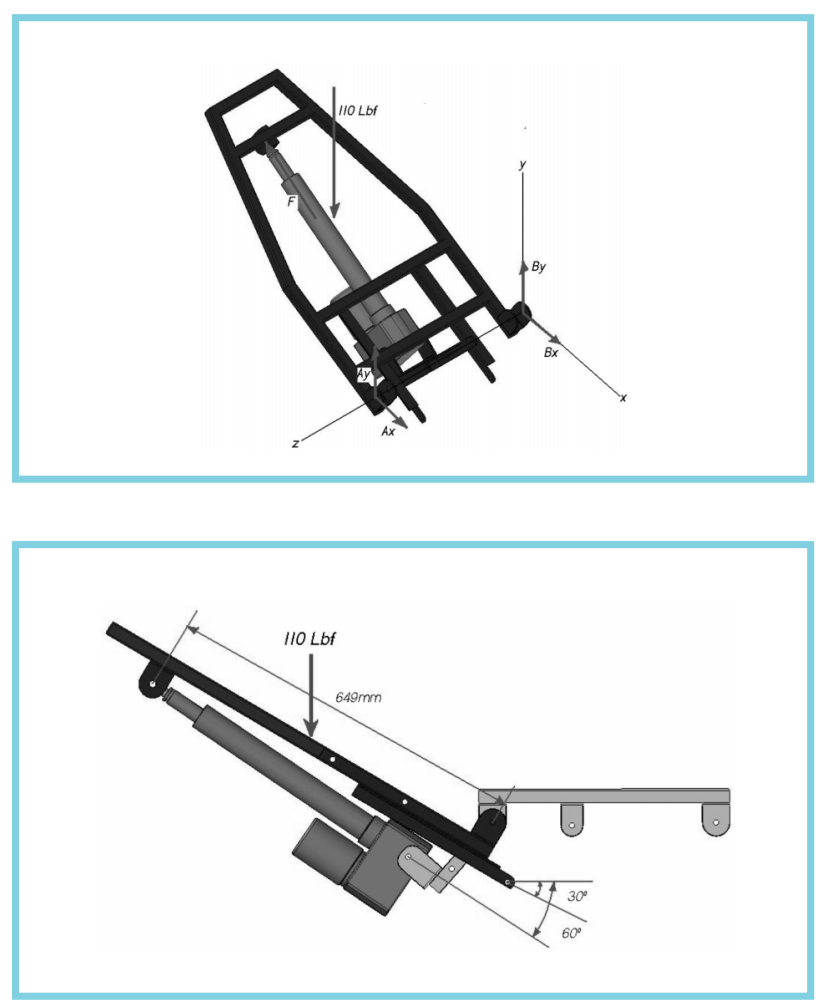

Figura 13. Sistema de reclinación.

Mediante el uso de diferentes dispositivos electrónicos que interactúan con el esqueleto mecánico a partir de un diseño mecatrónico, se implementa un sistema de control que permite el movimiento de la silla a voluntad de usuario.

Este sistema lo constituye una palanca de control o joystick, a la cual se le incorporaron un par de potenciómetros lineales, que se encargan de pensar la inclinación de dicha palanca. En los ejes de rotación tanto del sillín sobre el chasis, como del espaldar sobre el sillín, se insertan pasadores con una estría electro-erosionada con el fin de permitir en la unión entre cada una de las piezas la medición del ángulo de inclinación del sillín respecto del chasis y del espaldar respecto del sillín por medio de potenciómetros, como se observa en la figura 14.

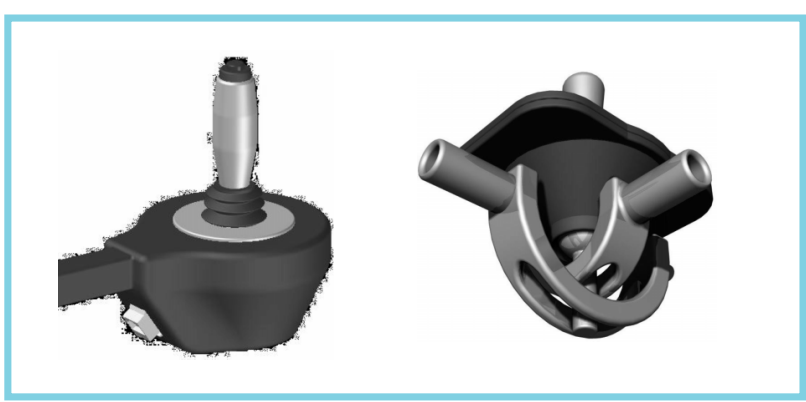

(a) Diseño del Joystick

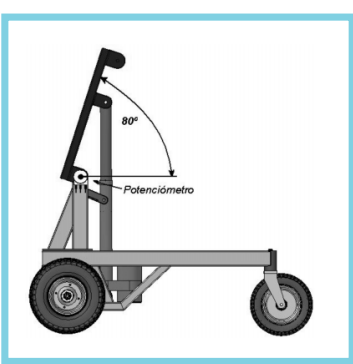

(b) Potenciómetro medición ángulo del chasis

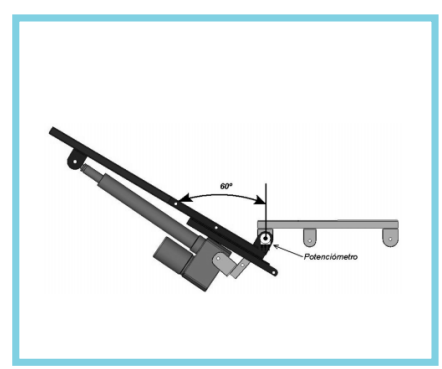

(c) Potenciómetro medición ángulo del espaldar
Figura 14. Diseño del sistema de medición de posición.

En la parte trasera de la silla de ruedas van apoyadas dos baterías de 12 voltios x 70 amperios cada una, conectadas en serie, con el fin de proveer la energía para activar los dispositivos de la silla de ruedas, como se observa en la figura 15. Adicionalmente, se incorporó al sistema el modulo de voz VOICE DIRECT II, que permite la utilización de 15 palabras para el control de la silla de ruedas, éstas son: Reclinar, Declinar, Luz, Freno, Pito, Adelante, Atrás, Izquierda, Derecha cuyas salidas se ingresan al microprocesador con las del tablero de control por medio de unas compuertas tipo OR.

En la figura 15 se observa el prototipo final de la silla de ruedas en sus diferentes posiciones, asiento normal, reclinación y declinación. 


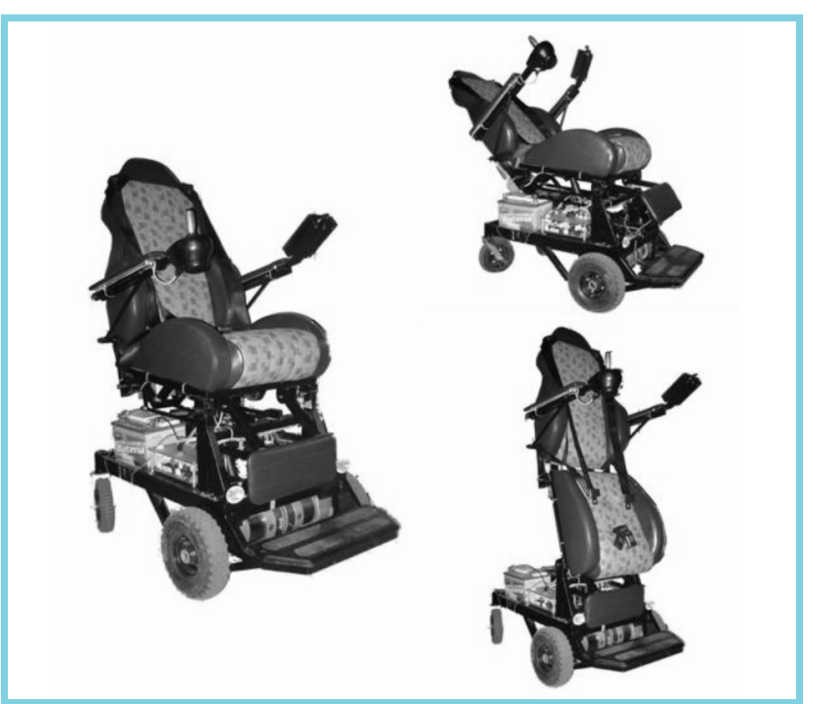

Figura 15. Sistema de reclinación.

Este desarrollo presenta una perspectiva del concepto de diseño mecatrónico, al desarrollar múltiples funciones para una silla de ruedas eléctrica. Adicionalmente, debido a sus dimensiones esta silla de ruedas se puede operar en lugares no diseñados para discapacitados, ya que puede transitar por corredores estrechos y puertas corrientes. Esta silla de ruedas cumple con los estándares de la ADA.

Al ser fabricada en acero cold rolled calibre 18, y al utilizar baterías de automóvil se aumenta su peso considerablemente, en este momento se está trabajando en cambios en el diseño, que involucran el estudio de nuevos materiales como aluminio, titanio, fibra de vidrio, fibra de carbono, y en la utilización de diferentes tipos de baterías (baterías secas o de gel), de tal forma que se pueda disminuir cuantiosamente su peso, factor importante a la hora de ser transportada en un vehículo.

\section{Diseño mecatrónico de manos robóticas}

El agarre humano posee una sorprendente flexibilidad e incomparable destreza, tal que ha llamado la atención de muchos investigadores.

El estudio del agarre humano como modelo ha llevado a la construcción de innovadores pero costosos prototipos de dedos. Ejemplos de referencia son las manos de Stanford/JPL hand (7), Utah/MIT hand (8), TUAT/Karlsruhe Humanoid hand (7), DLR Hand, (9), Robonaut hand de la Nasa (10). La primera versión de mano robótica realizada en la Universidad Militar Nueva Granada fue una mano antropomórfica de cuatro dedos que imita los modelos prensiles humanos el inconveniente es el elevado número de actuadores utilizados y el costo para su implementación (11).

Posteriormente se diseñó un prototipo de dedo antropomórfico con el tamaño similar al de un dedo índice humano que utiliza un menor número de actuadores, más económico y de funcionamiento sencillo comparado con la versión de mano anterior. La finalidad de este prototipo fue usarlo como módulo para el diseño de una mano robótica antropomórfica de cinco dedos. Para el diseño de este mecanismo se usó el software de simulación mecánica SolidWorks® y Visual Nastran® (12).

El análisis cinetostático del mecanismo fue resuelto utilizando la metodología de grupos de Assur, este proceso inicia con la solución gráfica obtenida con software de CAD, como se observa en la figura 16a. Para encontrar todas las posiciones de cada uno de los puntos de interés del mecanismo y para resolver el problema del cálculo de las fuerzas en los eslabones y los pasadores del dedo se utilizó software de simulación numérica, el Toolbox Assur v1.0 de Matlab®, además de simulaciones realizadas con software para el análisis de esfuerzos, Ansys ${ }^{\circledR}$, como se observa en la figura $16 \mathrm{~b}(13)$.

Teniendo en cuenta que la entrada del mecanismo es un movimiento lineal (emulando el par agonista/antagonista del sistema musculo/tendon para la generacion de un movimiento de flexion/ extension de un dedo), se utiliza como actuador un conjunto micromotor DC acoplado a un tornillo sin fin, como se muestra en la figura 16c. 


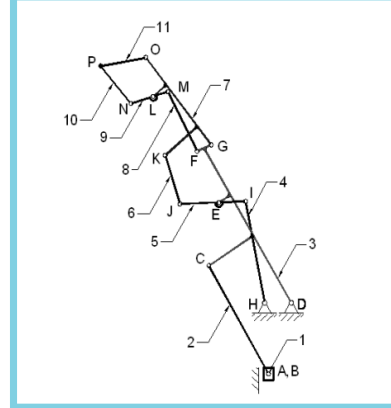

a) Sistema de palancas

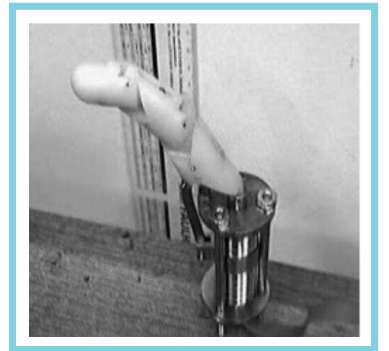

c) Ejemplos de posiciones alcanzadas

Figura 16. Dedo antropomorfico.

A partir del diseño del dedo se realizó el diseño mecatrónico de una mano con 5 dedos y una palma. Para conseguir los movimientos deseados se utiliza un sistema de palancas. Con base al estudio de los movimientos de prensión y manipulación de la mano se diseña un sistema de palancas para recrear la flexión y extensión del dedo humano. En total la mano posee 6 GDL, los dedos cuentan con 1 GDL (para extensión/ flexión), el pulgar posee 2 GDL abducción/aducción además de la flexión/extensión (14).

La estructura mecatrónica fue diseñada teniendo en cuenta la biomecánica de la mano $(1,6,16)$ y sus medidas antropomórficas para así definir las caracteristicas cinemáticas y dinámicas que permitan imitar tanto como sea posible el movimiento de la mano, como se observa en la figura 17.

El prototipo final tiene medidas antropomórficas y está diseñada para ser similar en apariencia y funcionalidad a una mano humana. Se realizan pruebas y simulaciones del desempeño funcional de la mano antropomórfica.

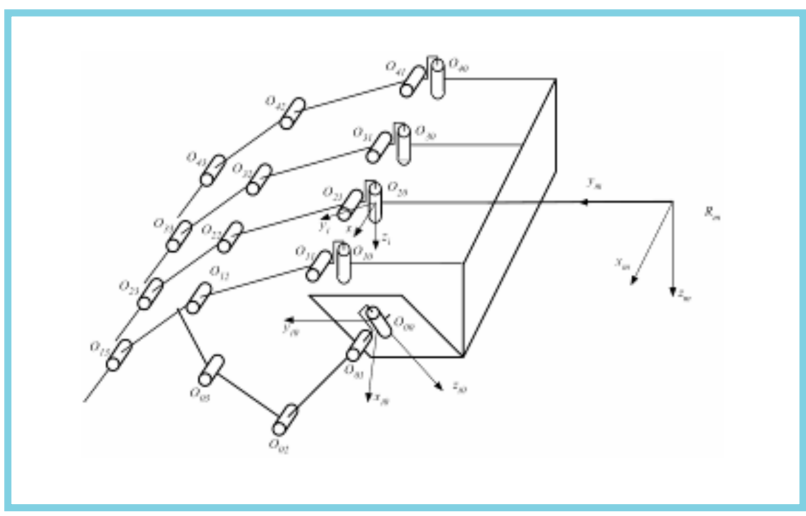

Figura 17. Modelado cinemático de la mano humana.

El estudio de la cinemática del mecanismo por medio del método gráfico permite obtener ecuaciones polinomiales que relacionan las trayectorias de cada articulación, posteriormente se realizó la simulación y de los resultados obtenidos se determinaron los siguientes parámetros para la mano MUC-I:

1. Ausencia de todos los grados de libertad en la muñeca (dado que la finalidad del diseño es acoplarla a un brazo robótico), los únicos elementos móviles son los dedos.

2. La articulación metacarpofalángica tiene un solo grado de libertad.

3. Las articulaciones distalinterfalángica y proximalinterfalángica, son de revolución (un grado de libertad).

En la figura 18 se observan los grados de libertad de la mano diseñada en CAD.

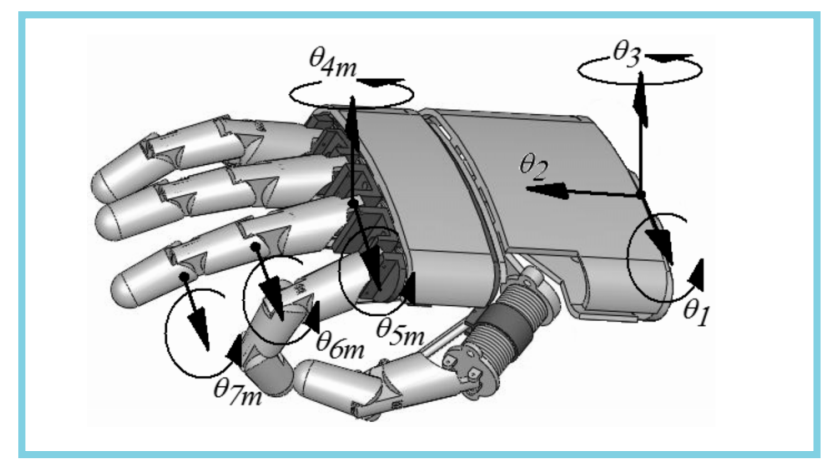

Figura 18. Modelo en CAD de la MUC-I. 
Se realizaron simulaciones de los mecanismos diseñados y del comportamiento de la mano al realizar diferentes tipos de agarre, con un programa desarrollado para ese propósito, como se observa en la figura 19.

Con el fin de validar el espacio de trabajo de los dedos de la MUC-I, se desarrolló un programa que permite verificar las trayectorias y el espacio de trabajo de cada dedo de la mano diseñada, en la figura 20 se presenta la interfaz de este programa (15).

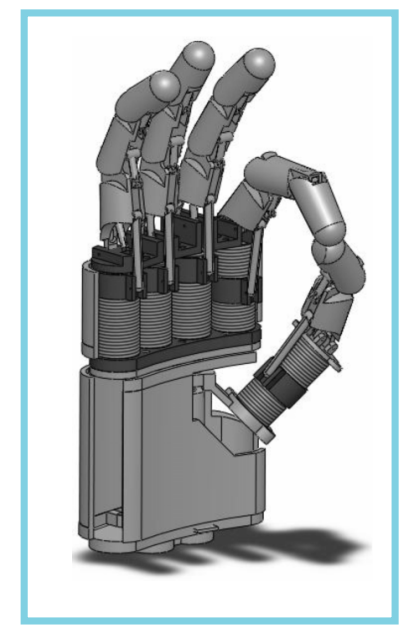

a) Vista frontal

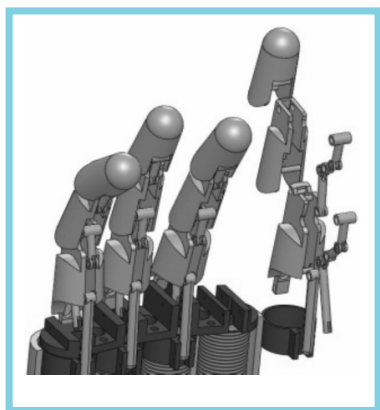

c) Vista detallada del dedo índice

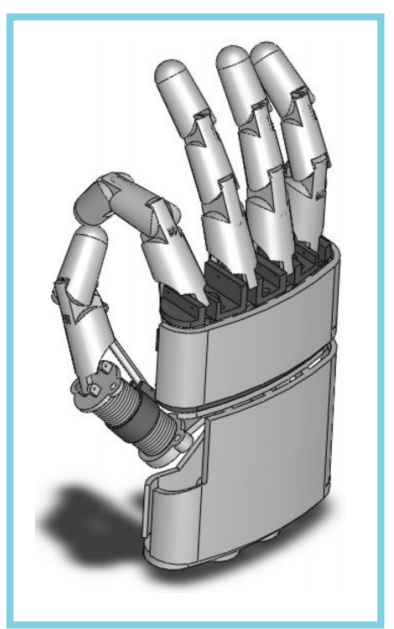

b) Vista posterior

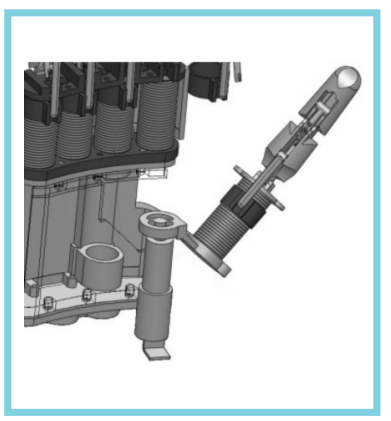

d) Detalle del pulgar
Figura 19. Mano mecánica MUC-I.

La MUC-I puede realizar movimientos prensiles y no-prensiles, ya que en la mano humana, la estabilidad es un requisito importante a la hora de aga- rrar un objeto, hay dos formas básicas de agarre, éstas son:

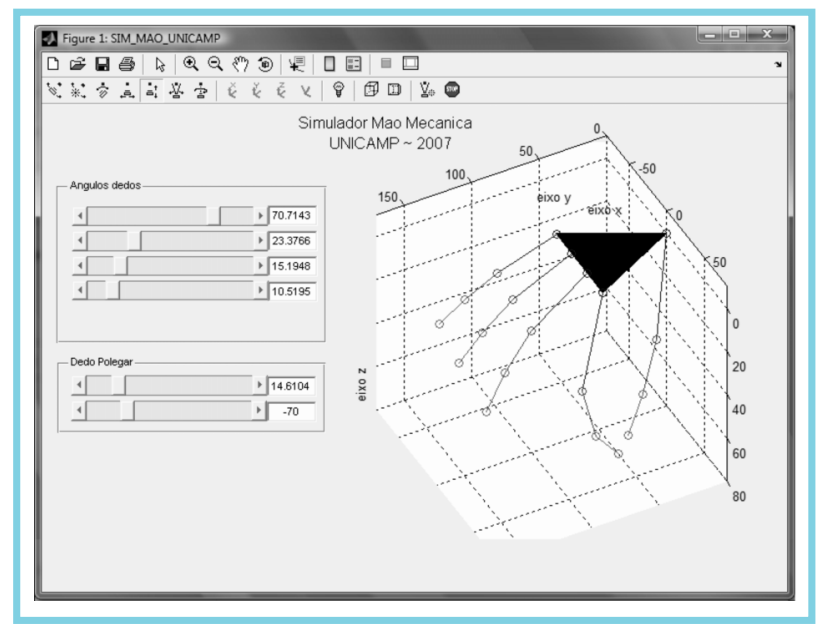

Figura 20. Programa para el análisis del espacio de trabajo de los dedos de la MUC-I.

- El agarre de poder, en el cual a través de la flexión de los dedos se sostiene el objeto contra la palma, como se observa en la figura $10 \mathrm{~b}$.

- El agarre de precisión, en el cual el objeto es asegurado con la punta de uno o más dedos con la punta del pulgar como se observa en la figura 10a.

En general la mano mecánica MUC-I fue desarrollada teniendo en cuenta la antropometría y funcionalidad de una mano humana, se proyectó y construyó un dedo de fabricación simple como elemento modular, lo cual facilitó el montaje final. El prototipo consta de cinco dedos, de los cuales, sólo el pulgar tiene dos grados de libertad, los demás tienen solo uno y esto le permite oponerse a la palma de la mano permitiendo realizar los agarres de prensión de potencia y de precisión. Se hace uso de técnicas de prototipado rápido para sistemas mecánicos para facilitar el montaje final de los mecanismos de barras, ya que este método permite obtener los conjuntos ya ensamblados. El uso de micromotores DC acoplados a los tornillos sin fin imita el comportamiento del conjunto de músculo-tendón en los cuales la función agonista 
y antagonista es representada por los giros del motor en un sentido u otro.

Para realizar las diferentes pruebas de operación la mano MUCI se implementó sobre un robot antropomórfico para ser utilizada como gripper industrial, figura 22.

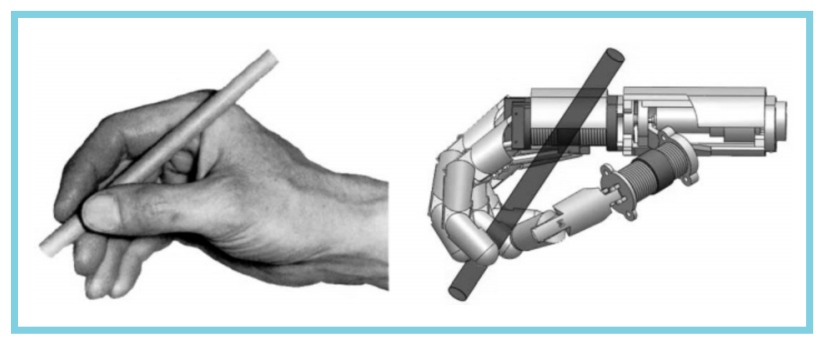

a) Agarre de precisión prismático

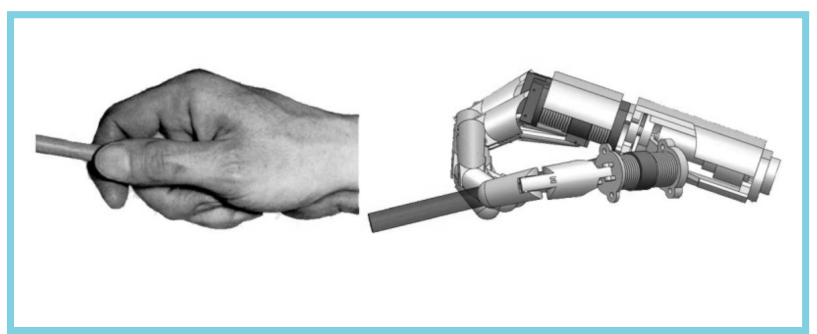

b) Agarre de potencia prensil

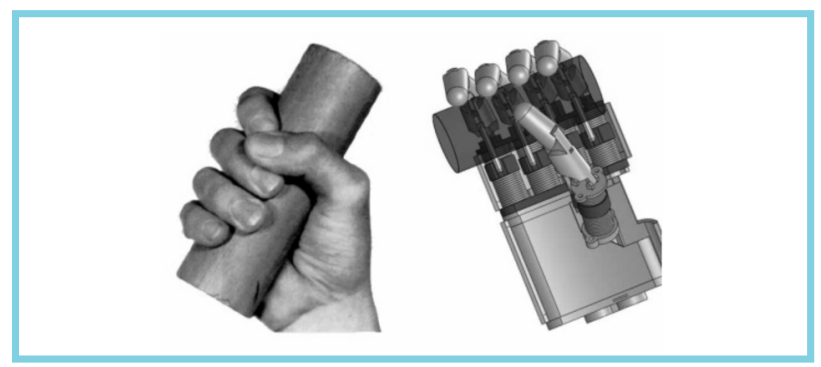

c) Agarre de potencia prensil prismático

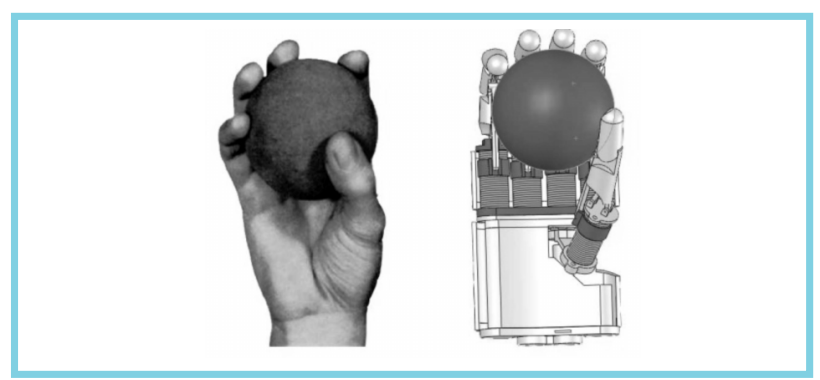

d) Agarre de potencia prensil circular

Figura 21. Imágenes de comparación entre los agarres humanos y los de la MUC-I.
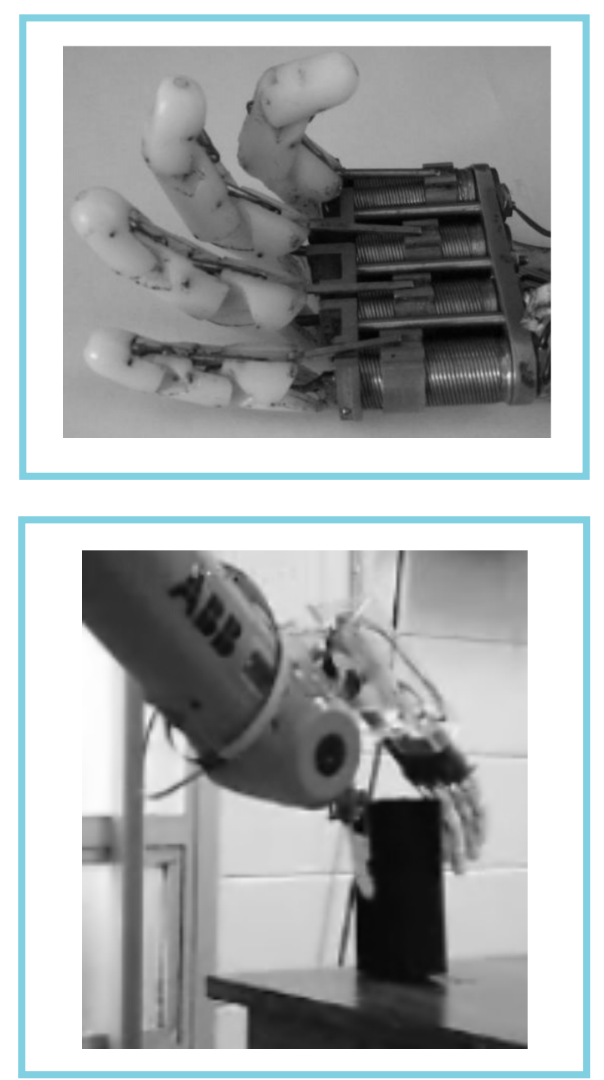

Figura 22. Prototipo final implantado en un brazo robótico.

\section{Conclusiones}

Se presentó una metodología de diseño para el desarrollo de dispositivos utilizados para solucionar algunas de las necesidades de personas con limitaciones físicas o mentales, ya sean temporales o permanentes.

El diseño mecatrónico permite partir de un modelo computacional para llegar a un prototipo virtual, que garantizara que el prototipo físico final cumplirá con los requerimientos establecidos, antes de ser implementado.

Esta metodología permite reducir los costos y el tiempo de desarrollo de nuevos productos, logrando consecuentemente una disminución en el costo de fabricación y en el costo final para el usuario. Lo cual es muy importante dado que en nuestro país la cantidad de personas con limitaciones 
físicas aumenta cada día y la mayoría son individuos de limitados recursos económicos.

Se presentaron tres diseños que permiten demostrar determinados desarrollos tecnológicos en el país.

Recibido: junio 2008

Aceptado: octubre 2008

\section{Bibliografía}

1. Gutiérrez R, Vanega, F, Niño P. Diseño y control de un exoesqueleto para rehabilitación motora en miembro superior. IFMBE Proceedings IV Latin American Conference on Biomedical Engineering 2007. Bioengineering Solution For Latin America Health, vol. 18. Nov. 2007.

2. Rahman T, Stroud S, Ramanathan R, Alexander M, Seliktar R, Harwin W. Consumer Criteria for an Arm Orthosis, University of Delaware. 1996

3. Kulczycki, P. Fuzzy Controller for Mechanical Systems, IEEE Transactions on Fuzzy Systems, 2000; 8 (5), 645-652.

4. Avilés OF, Sánchez PA, Niño - Suárez C, Cárdenas L. Silla de ruedas multifuncional Bidepestador. $8^{\circ}$ Congreso Iberoamericano de Ingeniería Mecánica CIBIM2007- Cusco, Perú, 23 al 25 de octubre, 2007.

5. Cooper RA. Rehabilitation engineering applied to mobility and manipulation. Medical Science Series. Institute of Physics Publishing, Bristol and Philadelphia, 1995.

6. Matthew T, Mason J. Kenneth Salisbury Jr. Robot hands and the mechanics of manipulation. The MIT Press Series in Artificial Intelligence. 1985 .

7. Jacobsen SC, Wood JE, Knutti DF and Biggers KB. The UTAH/MIT dextrous hand: work in progress. In Robot, Grippers, Springer-Verlag, Berlin, 1986.
8. Hand S, Schulz C, Pylatiuk A. New Ultralight Anthropomorphic Inst. of Applied Computer Science Research Center of Karlsruhe, Alemania.

9. Butterfass J, Hirzinger J, Knoch GJ. and Liu H. DLR's multisensory articulated hand In Proc. of the 1998 IEEE Int. Conf. on Robotics and Automation, Leuven, Bélgica. May 1998.

10. Lovchik CS and Diftler MA. The robonaut hand: a dextrous robot hand for space. In Proc. of the 1999 IEEE Int. Conf. on Robotics and Automation, Michigan. May 1999.

11. Avilés O, Niño P, Rondón J, Ocampo G. Diseño y construcción de una mano robot de cuatro dedos XII Latin American Congress on Automatic Control, XIICLAC Salvador, Bahía, Brasil. 3 al 6 de octubre 2006.

12. Avilés O, Niño P, Rondón J, Ocampo G. Diseño y construcción de un dedo para grippers robóticas. Revista Colombiana de Rehabilitación, vol. 6, No. 6. Oct. 2007.

13. Avilés O, Rosário JM, Uribe A, Niño P, Gutiérrez R. Antrhopomorphic Grippers Modellind, Analysis and Implementation International Journal of Factory Automation, Robotics and Soft Computing. 2009.

14. Avilés O, Rosário JM, Uribe A, De Melo G. Mechanical hand shape based control MUCI experience. Workshop Robocontrol 08, 3rd applied robotics and collabotative systems engineering with emphasis in Industrial Applications and Educational Environments Workshop. Bauru, Brasil 4 y 5 de diciembre de 2008.

15. Avilés O, Rosario OJ, Uribe Á, Niño P. Diseño de un sistema mecatrónico antropomórfico de cinco dedos. XIII Congreso Latinoamericano de Control Automático / VI Congreso Venezolano de Automatización y Control. 